Galilean telescope arrays in infrared region using substrate-mode holograms

This content has been downloaded from IOPscience. Please scroll down to see the full text. 1993 J. Opt. 24113

(http://iopscience.iop.org/0150-536X/24/3/003)

View the table of contents for this issue, or go to the journal homepage for more

Download details:

IP Address: 140.113.38.11

This content was downloaded on 28/04/2014 at 17:28

Please note that terms and conditions apply. 


\section{GALILEAN TELESCOPE ARRAYS IN INFRARED REGION USING SUBSTRATE-MODE HOLOGRAMS}

DER-CHIN SU, YI-KONG TSAI and YANG-TUNG HAUNG

KEY WORDS :

Galilean telescope array

Substrate-mode hologram
MOTS CLÉS :

Matrice de télescopes galiliens

Hologramme de modes de substrat
SUMMARY: Galilean telescope arrays using substrate-mode holograms are presented. They are manufactured the visible region, and designed for operation in the infrared region. And they are formed in a monolithic block and compact, so they are easily introduced into an optical system. In addition, they do not introduce a local inversion as that in the Kepler telescope arrays.
Etalonnage du télescope Galilien en infrarouge utilisant les hologrammes en mode substrat

RÉSUMÉ: Les étalonnages du télescope Galilien utilisant les hólogrammes en mode substrat sont décrits. Ils sont créés dans la zone visible, destinés à opérer dans la région infrarouge. Ils sont formes dans un morrobloc compact pour être facilement introduits dans un système optique. En plus, ils ne provoquent pas une inversion locale à l'opposé des étalonnages du télescope Kepplex.

\section{I. - INTRODUCTION}

It is important to use telescope arrays for local beam compressing and optical interconnects in digital optical information processing and parallel optical bus with an infrared source [1]. There are several papers $[2,3]$ related to the telescope arrays for a visible light source. They are not monolithic, so they are not easily introduced into an optical system. Some other papers $[4,5]$ reported the holographic lens manufactured with a visible light source can be used in the infrared region. But the papers related to the telescope arrays in the infrared region are very few. Based on the coupled wave theory [6], Galilean telescope arrays with substrate-mode hologram [5] are proposed. They are manufactured in the visible region, and can be performed in the infrared region. And they can be formed in a monolithic block, so they are easily aligned and mounted. In addition, they are more compact and do not introduce a local inversion.

\section{II. - PRINCIPLE}

The structure of the conventional Galilean telescope arrays which consist of one positive and one negative arrays is shown in figure 1 . The compression ratio of the channels is equal to the ratio of the

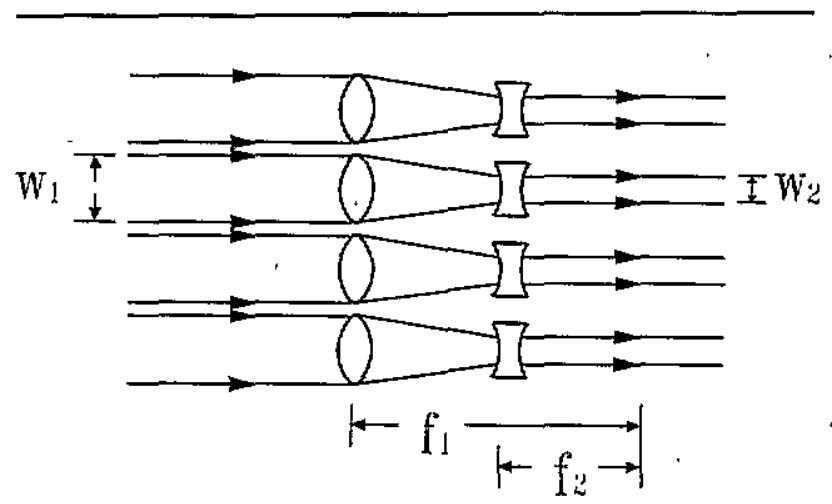

FIG. 1. - The configuration of the conventional Galilean telescope arrays. 


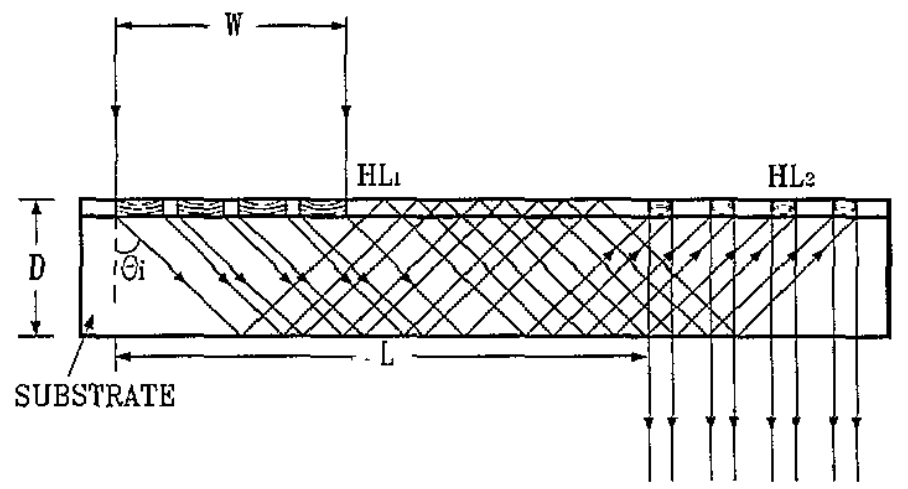

Eig. 2. - The schematic diagram of the Galilean telescope arrays using substrate-mode hologram.

$H L_{1}$ : input holo-lenslet array,

$H L_{2}$ : output holo-lenslet array.

focal length: $W_{1} / W_{2}=f_{1} /\left|f_{2}\right|$. If positive lenslet arrays are used instead of the negative lenslet arrays, they become the Kepler telescope arrays [7]. Comparing the Kepler and the Galilean telescope arrays, the Galilean are more compact and does not introduce a local inversion of channels. In this paper we discuss the Galilean type.

The schematic diagram of these Galilean telescope arrays with substrate mode hologram is shown in figure 2. It consists of two holo-lenslet array components $H L_{1}$ and $H L_{2}$ in a common substrate. Each component has $n$ (rows) $\times m$ (columns) hololenslets, and each holo-lenslet on the component has same characteristics. The holo-lenslets of $H L_{\mathrm{I}}$ diffract an incident global plane wave into local converging spherical waves which are guided into the underlying dielectric substrate at an angle exceeding the total internal reflection angle (i.e. critical angle). Then, they are guided along the design direction by reflecting off, or bouncing between, the upper and the lower planes of the substrate. After a moderate distance, they are recollimated by the holo-lenslets of $H L_{2}$ and propagate out of the substrate normally. The $i \times j$-th pair of holo-lenslet between $H L_{1}$ and $H L_{2}$ forms a channel, so there are $n \times m$ channels. The diffraction properties of a holo-lenslet placed at the origin of a right Cartesian coordinate can be summarized by the following equations [S]:

$$
\frac{1}{R_{i}}=\frac{1}{R_{r}} \pm \mu\left(\frac{1}{R_{o}}-\frac{1}{R_{c}}\right),
$$

and

$$
\sin \theta_{i}=\sin \theta_{r} \pm \mu\left(\sin \theta_{0}-\sin \theta_{c}\right) \text {. }
$$

Where $\mu=\lambda_{r} / \lambda_{c}$ is the ratio between the reconstruction and construction wavelength, $R_{q}(q=0, c, r, i)$ are the distances from these point sources of (object, reference, reconstruction, image) to the center of the holo-lenslet, $\theta_{q}$ denote the off-axis angles of the waves, and the \pm refer to +1 and -1 orders of the diffracted images of the holo-lenslet.

Because every holo-lenslet can be thought as a special kind of grating, hence the coupled wave theory [6] can be used to choose the optimal conditions for higher diffraction efficiency and high performances in the infrared region. The associated vectors of the first holo-lenslet for Bragg incidence is shown in figure 3 . In the figure, $k_{c}$ and $k_{o}$ are the wavevectors of the construction reference beam and the object beam at the wavelength $\lambda_{c,}$, and $k_{\mu}$ and $k_{i}$ are the wavevectors of the reconstruction beam and the diffracted beam at the wavelength $\lambda_{r}$, respectively. The magnitude of a wavevector is proportional to the reciprocal of wavelength, the magnitude of the wavevector of the recording beam is longer than that of the reconstruction beam. So, it is easy to find that the angle $\theta_{o}$ is smaller than the critical angle at the air-emulsion interface, but its corresponding diffraction angle $\theta_{i}$ is greater than the critical angle. The

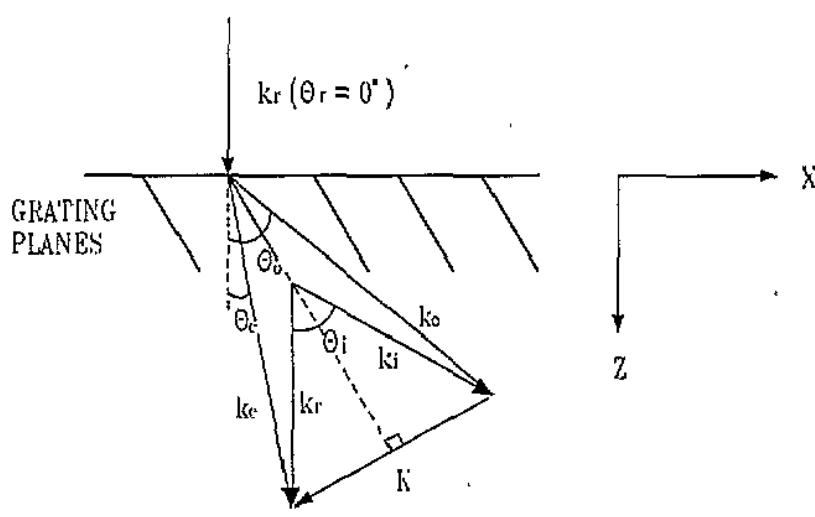

Fic. 3. - The K-vector diagram of a holo-lenslet for Bragg incidence. 


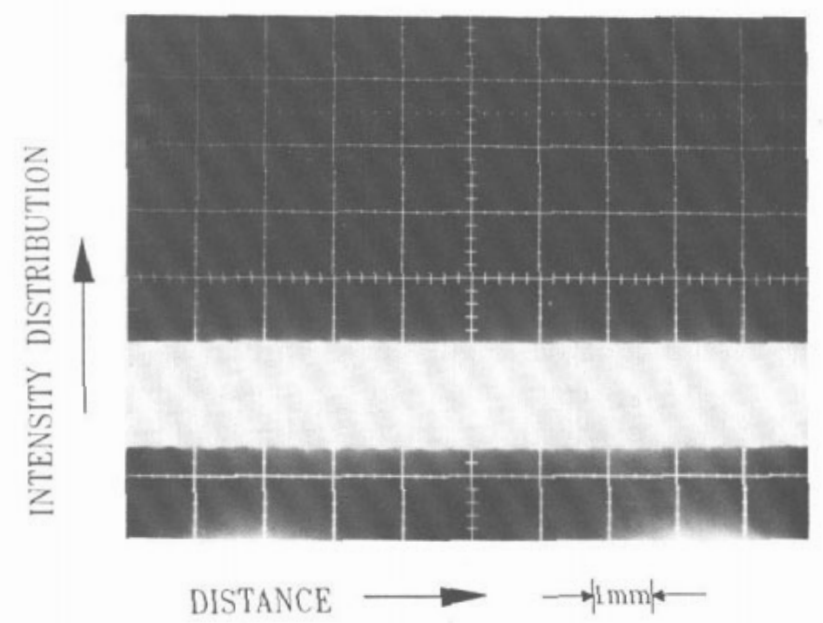

a)

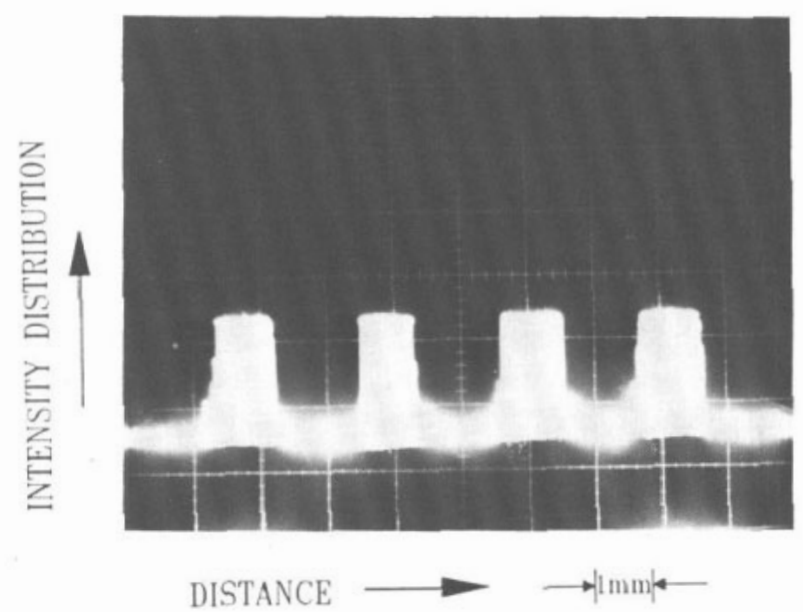

b)

\begin{tabular}{|c|c|c|c|}
\hline \multicolumn{2}{|c|}{ Sample } & 1 & 2 \\
\hline \multicolumn{2}{|c|}{$\begin{array}{c}\text { channel } \\
\text { compression ratio }\end{array}$} & $1 / 2$ & $1 / 4$ \\
\hline $\mathrm{HL}_{j}$ & $\begin{array}{c}R_{c^{\prime}}(\mathrm{mm}) \\
R_{n}(\mathrm{~mm}) \\
\theta_{a}(\mathrm{deg}) \\
\theta_{c}(\mathrm{deg}) \\
\text { diameter of } \\
\text { lenslet (mm) } \\
\text { lenslets } \\
\text { distance (mm) }\end{array}$ & $\begin{array}{c}\infty \\
-90.4 \\
13.1 \\
63.9 \\
2 \\
0.5\end{array}$ & $\begin{array}{c}\infty \\
-90.4 \\
13.1 \\
63.9 \\
2 \\
0.5\end{array}$ \\
\hline $\mathrm{HL}_{2}$ & $\begin{array}{c}R_{i}(\mathrm{~mm}) \\
R_{0}(\mathrm{~mm}) \\
\theta_{c}(\text { deg }) \\
\theta_{\mathrm{o}}(\text { deg }) \\
\text { dianeter of } \\
\text { lenslet }(\mathrm{mm}) \\
\text { lenslets } \\
\text { distance }(\mathrm{mm})\end{array}$ & $\begin{array}{c}-45.2 \\
\infty \\
63.9 \\
13.1 \\
1 \\
1.5\end{array}$ & $\begin{array}{c}-22.6 \\
\infty \\
63.9 \\
13.1 \\
0.5 \\
2\end{array}$ \\
\hline & $\begin{array}{l}\text { nce } L \text { (nm) } \\
\text { mber of total } \\
\text { eflection }\end{array}$ & $\begin{array}{l}20 \\
4\end{array}$ & $\begin{array}{c}30 \\
6\end{array}$ \\
\hline
\end{tabular}

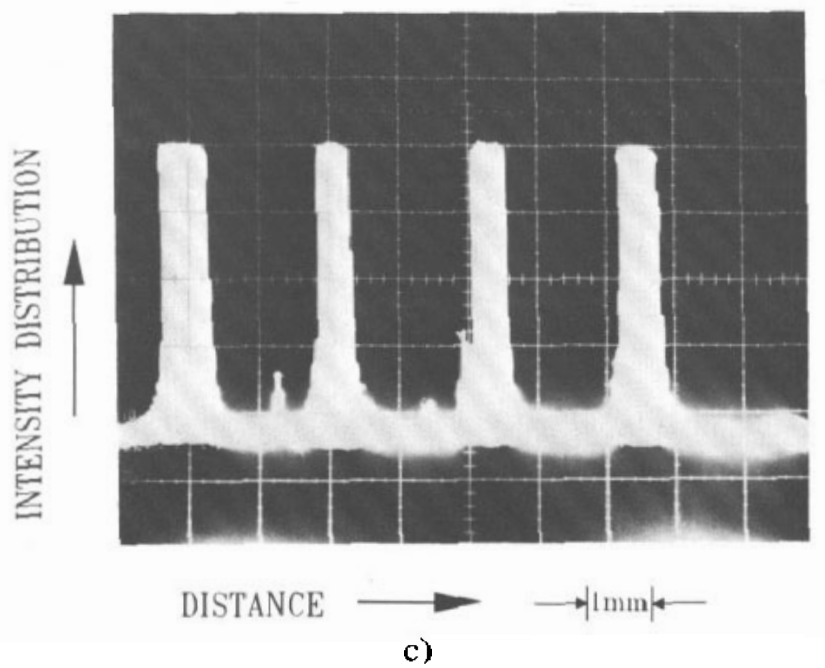

F1G. 4. - The intensity distribution of the input and output beams detected by an ascilloscope; (a) the input global plane beam ; (b) the output heams as compression ratio is $\frac{1}{2}$; (c) the output beams as compression ratio is $\frac{1}{4}$. 
holo-lenslet arrays $H L_{1}$ was designed for $\theta_{r}=0^{\circ}$ and $\theta_{i}=45^{\circ}$. Consequently, the output holo-lenslet $H L_{2}$ has $\theta_{r}=45^{\circ}$ and $\theta_{i}=0^{\circ}$. Two sets of Galilean telescope arrays were made by means of a step-andrepeat stup [3], and their fabrication parameters are listed in table 1 . They have $1 \times 4$ channels, and their sizes are $10 \mathrm{~mm} \times 40 \mathrm{~mm} \times 5 \mathrm{~mm}$ (thickness). Because the performance wavelength is different from the recording wavelength, there will be some aberrations. The aberrations are calculated with the formulae presented by Meier [10], and they are $2 \lambda$ and $3 \lambda$, respectively. To see the performances of these telescope arrays at $780 \mathrm{~nm}$, the intensity distributions of the input and the output beams detected by an oscilloscope are shown in figure 4. Figure $4 a$ shows the input global plane wave, and figure $4 b$ and figure $4 c$ show the output beams as the compression ratio $1 / 2$ and $1 / 4$, respectively. From these oscillograms, it is seen that the insertion loss is about $0.8 \mathrm{~dB}$, the widths of the output beam are $1.0 \mathrm{~mm}$ and $0.6 \mathrm{~mm}$, respectively.

\section{IV. - DISCUSSION AND CONCLUSTON}

The aberrations introduced by the difference between the recording wavelength and the reconstruction wavelength will mainly influence the qualities (e.g., the compression ratio and intensity distribution) of these telescope arrays. If the aspherical waves are used in the recording procedures [11], the aberrations will be deduced and the qualities of these telescope arrays will be improved. The telescope arrays for $780 \mathrm{~nm}$ are designed and manufactured, the telescope arrays for other wavelengths can also be manufactured with the same technique.

In this paper, the Galilean telescope arrays with substrate-mode hologram are proposed. They are manufactured in the visible region, and performed in the infrared region. Also they can be formed in a monolithic block, and easily introduced in an optical system. In addition, they are compact and do not introduce a local inversion.

\section{ACKNOWLEDGEMENT}

The research was supported by the National Science Council of ROC under contract NSC 81-0417E-009-562.

\section{REFERENCES}

[1] Lohmann AW. What Classical Optics can do for the Digital Optical Computer. Appl Opt, 1986;25:1543-1549.

[2] Tsai YK, Su DC, Yuang YT. A Holographic Lenslet Array Device with Variable Focal Power. Will appear in Optik.

[ 3] Lohmann AW, Sauer F. Holographic Telescope Arrays. Appl Opt, 1988; $27: 3003-3007$.

[ 4] Kostuk RK, Kato M, Huang XT. Polarization properties of substrate-mode holographic interconnects. Appl Opt, $1990 ; 29: 3848-3854$.

[ 5] Huang YT, Su DC, Tsai YK. Wavelength-Division-Multiplexing and Demultiplexing by using a Substrate-Mode Grating Pair. Opt Lett, $1992 ; 15: 1629-1631$.

[ 6] Kogelnik H. Coupled Wave Theory for Thick Hologram Gratings. Bell Sys Tech J, $1969 ; 48: 2909-2947$.

[ 7] Buralli DA, Morris M. Design of Two- and Three-Element Diffractive Keplerian Telescopes. Appl Opt, 1992; 31 : 38-43.

[ 8] Latta JN. Computer-Based Analysis of Hologram Imagery and Aberrations. I. Hologram Types and Their Nonchromatic Aberrations. Appl Opt, 1971; $10: 599-608$.

[ 9] Georgekutty TG, Liu HK. Simplified Dichromated Gelatin Hologram Recording Process, Appl Opt, 1987; 26:372376.

[10] Meier RW. Magnification and Third-order Aberrations in Holography. I Opt Soc Am, 1965; 55 : 987-992.

[11] Winick KA, Fienup JR. Optimum Holographic Elements Recorded with Nonspherical Wave Fronts, $J$ Opt Soc Am, I983; $73:$ 208-217.

(Manuscript received in April 6th 1993.) 